\title{
Pre-treatment serum lactate dehydrogenase (LDH) and serum alkaline phosphatase (ALP) as prognostic factors in patients with osteosarcoma
}

\begin{abstract}
Background: Osteosarcoma is a highly malignant primary bone tumour. The evaluation of prognostic factors influencing the survival rate is extremely important for defining the management approach of osteosarcoma patients. In this study, we evaluated the prognostic values of several factors and their influence on the final survival rate.

Method: 163 patients with high-grade conventional osteosarcoma of the extremity who were treated in a single institution between 2005 and 2010 were evaluated. Serological markers of pre-treatment serum lactate dehydrogenase (LDH) and alkaline phosphatase (ALP), as well as, age, gender, race, primary site of tumour, presence of metastasis, timing of metastasis and type of surgery were analysed.

Results: In our pool of patients, there was no statistical significance when comparing the survival rates in regards to age, gender, race, primary site of tumour and timing of metastasis.

We observed that the pre-treatment serum LDH and ALP, the presence of metastasis and type of surgery were statistically significant, independently, in the univariate statistical analysis. The 5-year overall survival rate of patients in the normal pre-treatment LDH group is $66.2 \%$; whereas in the high pre-treatment LDH group, it is $17.4 \%$. The 5 -year overall survival rate of patients in the normal pre-treatment ALP group is $55.8 \%$; whereas in the high pre-treatment ALP group, it is $26.7 \%$. In the multivariate statistical analysis, only the pre-treatment serum LDH and the presence of metastasis had maintained their prognostic significance since both the pre-treatment serum ALP and the type of surgery had lost their independent predictive value.

Conclusion: Based on the result, we could suggest that pre-treatment serum LDH and ALP would help to prognosticate osteosarcoma patient. Close follow-ups and regular evaluations are important for this group of patients to detect early systemic failure for salvage chemotherapy.
\end{abstract}

Keywords: osteosarcoma, prognostic, alkaline phosphatase, lactate dehydrogenase
Volume 9 Issue 2 - 2018

\author{
Y Sahran, ' Azlan Mohd Sofian, ${ }^{3}$ Arman \\ Zaharil Mat Saad, ${ }^{2}$ Wan Zulmi,' MZ Nor \\ Azman,' WI Faisham' \\ 'Department of Orthopaedics, School of Medical Sciences, \\ Health Campus, Universiti Sains Malaysia, Malaysia \\ ${ }^{2}$ Plastic Surgery and Reconstructive Sciences Unit, School of \\ Medical Sciences, Health Campus, Universiti Sains Malaysia, \\ Malaysia \\ ${ }^{3}$ Department of Orthopaedics, Sungai Buloh Hospital, Jalan \\ Hospital, Malaysia
}

Correspondence: Y Sahran, Department of Orthopaedics, School of Medical Sciences, Health Campus, Universiti Sains Malaysia, Kubang Kerian, Kelantan, Malaysia,Tel + 6097676398 , Fax+609 7676389, Email: sahran@usm.my

Received: July 7, 2017 | Published: March 20, 2018

\section{Introduction}

Osteosarcoma is a highly malignant primary bone tumour. It is a primary malignant tumour derived from primitive bone forming mesenchymal tissue, which is characterised by the production of osteoid or immature bone by the malignant proliferating spindle cells. It most commonly affects the adolescent and childhood age groups, and has a strong predilection for around the knee region. ${ }^{1}$

Despite the recent advancement in chemotherapy and improved prognosis, $30-40 \%$ of patients still succumb to the disease. ${ }^{1-4}$ Thus, the evaluation of prognostic factors influencing the survival rate is extremely important for the management approach of patients. ${ }^{4}$ Adequate information may provide better counselling sessions and good patient-physician relationships, which will improve compliance to the treatment. ${ }^{5,6}$

There was a review article regarding the prognostic factors in non-metastatic osteosarcoma of the extremities, which was based on the analysis of eight reports published between 1973 and 1992. The conclusion was the only proven prognostic variable for patients with osteosarcoma of the extremities was the rate of tumour necrosis induced by pre-operative chemotherapy. ${ }^{7,9}$ Chemotherapy response as a prognostic indicator has been consistently reported, however, various studies on other prognostic variables that may be used to stratify patients before the onset of therapy yielded different results. ${ }^{7-11}$

Information on chemotherapy response only known after treatment has begun. Availability of prognostic factors before treatment will be very helpful for the decision-making and counselling of osteosarcoma patients. Ideally, chemotherapy and a surgery regimen could be tailored for individual patients according to their disease prognosis.

In a systemic review of prognostic factors in localised extremity osteosarcoma, 93 papers were extensively studied. Although the literature is abundant, only a few papers are of sufficient quality to allow solid conclusions. Several "new" prognostic factors seem to be independent factors; chemotherapy response, tumour size and site, alkaline phosphatase level and p-glycoprotein expression. ${ }^{9,11}$

Several studies were accomplished to evaluate biochemical markers as prognostic factors. However, based on the available literatures, the prognostic value of ALP and LDH serum levels remain controversial until today. ${ }^{8}$ 
An international collaboration with the combined total of 2680 patients were examined concerning the prognostic factors of osteosarcoma, yet the role of LDH and ALP were not analysed. ${ }^{10}$

The latest evidence on the prognostic significance of LDH levels in osteosarcoma was published in a meta-analysis, which combined 10 studies between 1997 to 2013 from 9 countries. The analysis included 943 patients and showed the statistically significant role of the serum LDH level on the overall survival of osteosarcoma patients. ${ }^{8}$ The same report hadn't analysed the role of ALP.

The glycolytic enzyme lactate dehydrogenase (LDH) is a biological marker for cytosol in various tissues, and its serum levels are high in numerous pathological conditions. In bone tumours, serum LDH has been found helpful as a prognostic factor, and for evaluating the response of treatment in patients with Ewing's sarcoma. ${ }^{9}$ The possible correlation between serum LDH levels and the prognosis of osteosarcoma has been investigated in a small number of clinical studies. ${ }^{9,10,12}$

Alkaline phosphatase (ALP) is a non-specific enzyme that can hydrolyse a variety of phosphates in alkaline hydrolysis conditions. It is a membrane metal glycoprotein, formed by four isozymes. Many pathological conditions or diseases cause different isozyme elevations, thus increasing the total activity of ALP. ${ }^{13}$

The aim of our study is to determine the value of serum alkaline phosphatase (ALP) and lactate dehydrogenase (LDH) for predicting prognosis and overall oncological outcome in patients with osteosarcoma. We analysed the prognostic factors (particularly ALP and LDH levels) before treatment began, as well as, other general variables including age, gender, race, and primary site of tumour and timing of metastasis.

\section{Materials and methods}

Ethical approval from the School of Medical Science Research \& Ethics Committee, Hospital Universiti Sains Malaysia (HUSM) was obtained prior to the commencement of this study. A retrospective review was performed on the medical records of osteosarcoma patients who were treated in HUSM between January 2005 and December 2010. We included all patients who were newly diagnosed or with previously untreated osteosarcoma. We studied only patients with histologically confirmed high-grade conventional osteosarcoma through electron microscopy evaluation and immunohistochemistry by pathologist(s). We excluded osteosarcoma involving the axial skeleton, mandible, skull, soft tissue osteosarcoma, surface lesions and other osteosarcoma subtypes. We also excluded patients with doubtful histological diagnoses, patients whose deaths were due to chemotherapy complications and patients with insufficiently recorded clinical data.

All patients with a diagnosis of osteosarcoma treated at our centre were identified through the medical record unit database, and all case folders were retrieved. Initially, 192 patients were identified for this study. After the selection following the exclusion criteria, 163 patients were available for evaluation. A total of 163 patients (109 males, 54 females) with the mean age of 18.9 years were reviewed.

The patients' serum levels of ALP and LDH were recorded from the case notes or computer system. All 163 patients had the ALP and LDH values measured during the initial assessment. Serum ALP (IU/L) was determined using Dimensions ALPI method (Siemens,
Munich, Germany). Serum LDH, reported in International Units per Litre (IU/L), was determined using the Dimensions LDI method (Siemens, Munich, Germany).

The normal range of serum ALP was 53 to $168 \mathrm{IU} / \mathrm{L}$ for males; while in females, the normal range was 42 to $98 \mathrm{IU} / \mathrm{L}$. Levels higher than $168 \mathrm{IU} / \mathrm{L}$ in males and $98 \mathrm{IU} / \mathrm{L}$ in females were considered pathologically high. The normal range of serum LDH is 225 to 450IU/L, and serum levels lower than 450IU/L are considered normal. Levels higher than 450IU/L are considered pathologically high.

We evaluated the pre-treatment serum LDH and ALP levels and its correlation with the clinical prognostic value. Serum activity of LDH and ALP was estimated by the standard method of our centre's chemistry laboratory. The normal range of serum LDH is 225 to 450 IU/L. Levels higher than $450 \mathrm{IU} / \mathrm{L}$ are considered pathologically high. The normal range of serum ALP is 53 to $168 \mathrm{IU} / \mathrm{L}$ in males, and 42 to 98IU/L in females. Levels higher than 168IU/L in males and 98IU/L in females are considered pathologically high.

Other general variables for prognosis were also recorded, including patients' age at the time of presentation, gender, primary tumour site, histological type and extent of disease. Extent of disease is defined as the involvement of metastases, either at presentation, during or after treatment. Types of surgical intervention and follow-up duration were also recorded.

\section{Statistical analysis}

Event Free Survival (EFS) was measured from the date of diagnosis to the date of disease progression, recurrence, second malignancy or death from any cause, whichever occurred first; or to the date of the last follow-up for patients without any events. Overall Survival (OS) was measured from the first date of diagnosis to the date of death from any cause or last follow-up date.

Survival data and statistical analysis was done using STATA 11.2. This study applied survival analysis which concerns time to event data using phenotypic and biological prognostic factor and outcome (EFS and OS) variables, as previously defined. Data were entered and assessed using SPSS version 20.0. The distribution and completeness of data on the variables under study were observed. The percentages for categorical variables (such as age, gender, site of tumour, presence of metastases) and the mean with standard deviation for numerical data (LDH, ALP) were calculated.

Univariate Analysis, Median OS and 5-year EFS were calculated using Kaplan Meier Survival Curve. Survival probabilities across groups were calculated and plotted for each variable using Kaplan Meier. Log Rank Test was employed to compare the survival rate for all categories of independent variables, followed by pair wise multiple comparison if they were more than 2 groups.

Univariate Cox Hazard Regression analysis was calculated for all independent variables to obtain the preliminary data of which independent variable had prognostic value for the survival outcome of our osteosarcoma patients.

Multivariable Analysis and Multiple Cox Hazard Regression was applied to identify the independent prognostic factors with multiple co-variates. All variables with $p<0.25$ in the univariate Cox Hazard Regression analysis, and other variables that are clinically and biologically important, were included. 
Survival plots were generated using the Kaplan-Meier actuarial survival method and statistical significance derived from the log-rank test. These survival analyses, along with Cox proportional hazards models, were applied for the univariate analysis.

Multivariate analysis was also generated using the Cox proportional hazards regression model, with significance obtained from the Wald statistic. Statistical significance was defined as $\mathrm{p}<0.05$ and inclusion in the multivariate model was restricted to variables with a univariate significance of $\mathrm{p}<0.20$.

\section{Results and discussion}

A total of 163 patients (109 males, 54 females) were reviewed Patients' mean age was 18.9 years, ranging from 6 to 59 years old. The mean follow-up was 40.2 (36-84 months). Limb salvage surgery was performed in $80(49.1 \%)$ patients, and $41(25.2 \%)$ underwent amputation. However, 46 patients $(28.2 \%)$ did not undergo any surgical intervention and did not complete chemotherapy.

The disease-free survival of 163 patients was 40\% (Figure 1). 117 patients who completed both chemotherapy and surgical treatment had the survival rate of $71.8 \%$ at 5 years. Patients who did not complete treatment had $13 \%$ survival rate at 5 years. Based on the multivariate analysis, pulmonary metastases and completeness of treatment have a significant, negative impact on survival (Table 1).

The mean pre-treatment LDH level was $493.2 \mathrm{mmol} / \mathrm{L}$ and the ALP was $272 \mathrm{mmol} / \mathrm{L}$. In the normal pre-treatment LDH group, the overall survival rate at 5 years is $66.2 \%$; whereas in the high pretreatment LDH group, survival rate at 5 years is $17.4 \%$ (Figure 2). The overall survival rate in the normal pre-treatment ALP group is $55.8 \%$ at 5 years; whereas in the high pre-treatment ALP group, it is $26.7 \%$ (Figure 3 ). Both values were statistically significant with the univariate analysis. In the multivariate statistical analysis, only the pre-treatment serum LDH and presence of metastasis maintained their prognostic significance since both the pre-treatment serum ALP and the type of surgery had negative, independent predictive values.

There was no significant difference in the survival rates according to gender, age and site of tumour (Table 2), (Table 3).

Table I Prognostic factors of Osteosarcoma by Univariable and Multivariable Cox Proportional Hazards Models

\begin{tabular}{|c|c|c|c|c|c|c|}
\hline \multirow[b]{2}{*}{ Variables } & \multicolumn{3}{|c|}{ Simple Cox regression } & \multicolumn{3}{|c|}{ Multiple Cox regression } \\
\hline & (b) & Crude HR (95\%CI) & p-value & (b) & Adjusted HR (95\%CI) & p-value \\
\hline \multicolumn{7}{|l|}{ Surgery } \\
\hline LSS & 0.00 & 1.00 & - & 0.00 & 1.00 & - \\
\hline Amputation & 0.62 & $1.87(1.22,2.85)$ & $0.004 *$ & 0.34 & $1.41(0.32,6.23)$ & 0.66 \\
\hline \multicolumn{7}{|l|}{ Metastasis } \\
\hline Localized & 0.00 & 1.00 & - & 0.00 & 1.00 & - \\
\hline Metastatic & 1.45 & $4.25(2.77,6.50)$ & $<0.001 *$ & 1.04 & $2.82(1.70,4.70)$ & $<0.001 *$ \\
\hline \multicolumn{7}{|l|}{ LDH } \\
\hline Normal & 0.00 & 1.00 & - & 0.00 & 1.00 & - \\
\hline High & 1.54 & $4.64(2.94,7.35)$ & $<0.001 *$ & 1.20 & $3.32(1.85,5.96)$ & $<0.001^{*}$ \\
\hline \multicolumn{7}{|l|}{ ALP } \\
\hline Normal & 0.00 & 1.00 & - & 0.00 & 1.00 & - \\
\hline High & 0.99 & $2.71(1.77,4.14)$ & $<0.001 *$ & 0.40 & $1.49(0.85,2.61)$ & 0.17 \\
\hline
\end{tabular}

Backward stepwise Cox proportional hazard regression model applied.

No interaction in the model. Log-minus-log plot and hazard function plot were applied to check the model assumption and found fulfilled.

Table 2 Association between survival status and biological markers

\begin{tabular}{llcll}
\hline Variables & \multicolumn{2}{l}{$\begin{array}{l}\text { Survival Status } \mathbf{n}(\%) \\
\text { Died }\end{array}$} & OR (95\%Cl) & p-value* \\
\hline LDH & Alive & 26(33.8) & $9.29(4.47,19.27)$ & $<0.00 I^{* *}$ \\
Normal & $51(66.2)$ & $71(82.6)$ & & \\
High & $15(17.4)$ & & & \\
ALP & $43(55.8)$ & $34(44.2)$ & & $<0.00 I^{* *}$ \\
Normal & $23(26.7)$ & $63(73.3)$ & $3.46(1.80,6.68)$ & \\
High & & & &
\end{tabular}


Table 3 Association between survival status and prognostic factors

\begin{tabular}{|c|c|c|c|c|}
\hline \multirow[t]{2}{*}{ Variables } & \multicolumn{2}{|c|}{ Survival Status n (\%) } & \multirow[t]{2}{*}{ OR $(95 \% \mathrm{CI})$} & \multirow[t]{2}{*}{ p-value* } \\
\hline & Alive & Died & & \\
\hline \multicolumn{5}{|l|}{ Gender } \\
\hline Female & $23(42.6)$ & $3 I(57.4)$ & \multirow[b]{2}{*}{ I. $14(0.59,2.21)$} & \multirow[b]{2}{*}{0.7} \\
\hline Male & $43(39.4)$ & $66(60.0)$ & & \\
\hline \multicolumn{5}{|l|}{ Age } \\
\hline$<18$ & $44(43.6)$ & $57(56.4)$ & I.4(0.73,2.70) & 0.31 \\
\hline$>18$ & $22(35.5)$ & $40(64.5)$ & & \\
\hline \multicolumn{5}{|l|}{ Metastasis } \\
\hline Localized & $4 I(56.2)$ & $32(43.8)$ & $3.33(1.73,6.40)$ & $<0.001 * *$ \\
\hline Metastasis & $25(27.8)$ & $65(72.2)$ & & \\
\hline \multicolumn{5}{|l|}{ Surgery } \\
\hline LSS & $45(56.8)$ & $35(43.2)$ & $6.15(2.92,12.93)$ & $<0.00 I^{* *}$ \\
\hline Amputation & $7(17.6)$ & $34(82.4)$ & & \\
\hline
\end{tabular}

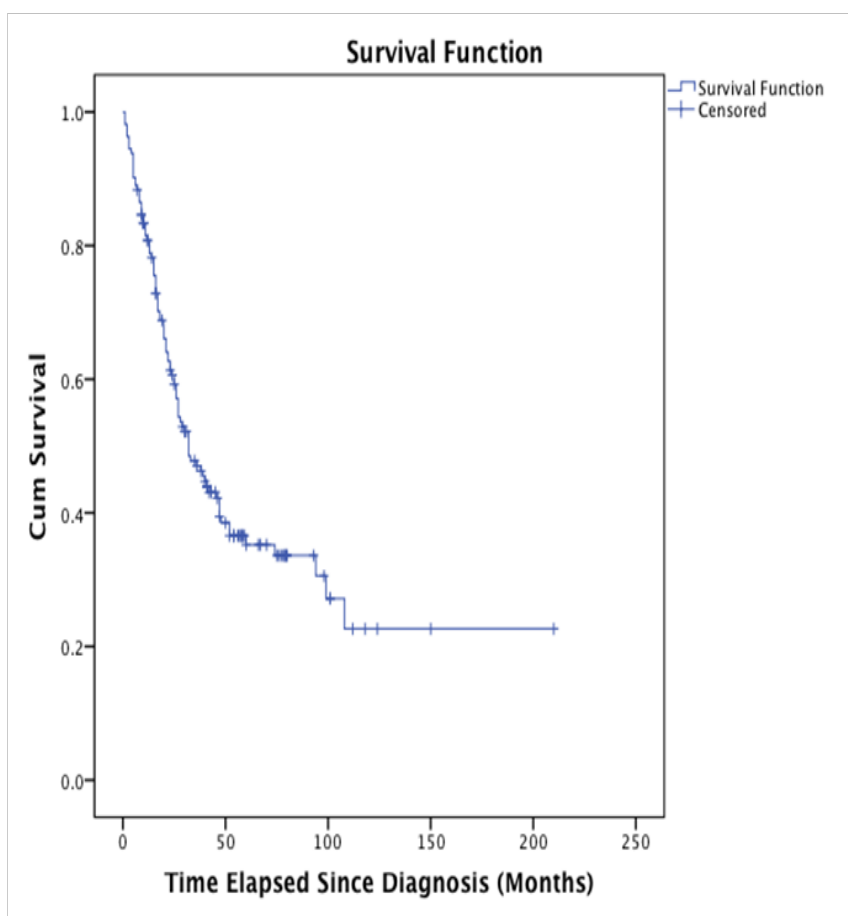

Figure I Overall survival of Osteosarcoma patients in our study.

In our group of patients, the mean LDH level was 493.19(IU/L) with standard deviation of 598.00. The mean ALP level was 272.93(IU/L) with standard deviation of 437.00 .

The overall survival, calculated from the initial presentation, to the time of death or the last follow-up, was determined according to the Kaplan-Meier product limit estimate. Differences between groups were compared by means of the log-rank test. In the multivariate analysis, the prognostic variables were evaluated using the Cox regression analysis.

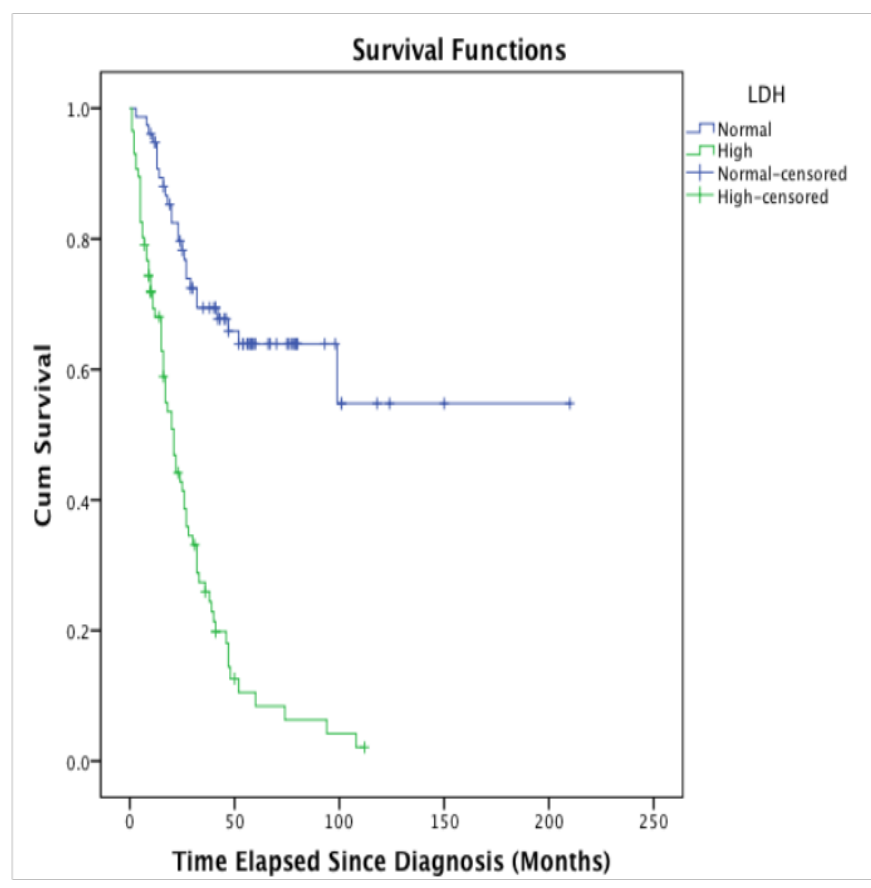

Figure 2 Overall survival of Osteosarcoma with high LDH.

There is a significant association between LDH and the survival status using Pearson Chi-square test. We observed a higher number of patients that succumbed to the disease with elevated levels of LDH $(82.6 \%)$. Those who passed away were 9.3 times at greater odds of being in the high-level LDH group compared to those in the surviving group.

Similar to the biological marker of $\mathrm{LDH}$, there is a significant association between ALP and the survival status using Pearson Chi-square test. We observed that a greater number of patients that succumbed to the disease had high levels of ALP (73.3\%). Those 
who passed away were 3.5 times at increased odds of being in the high-level ALP group compared to those in the group of patients that survived.

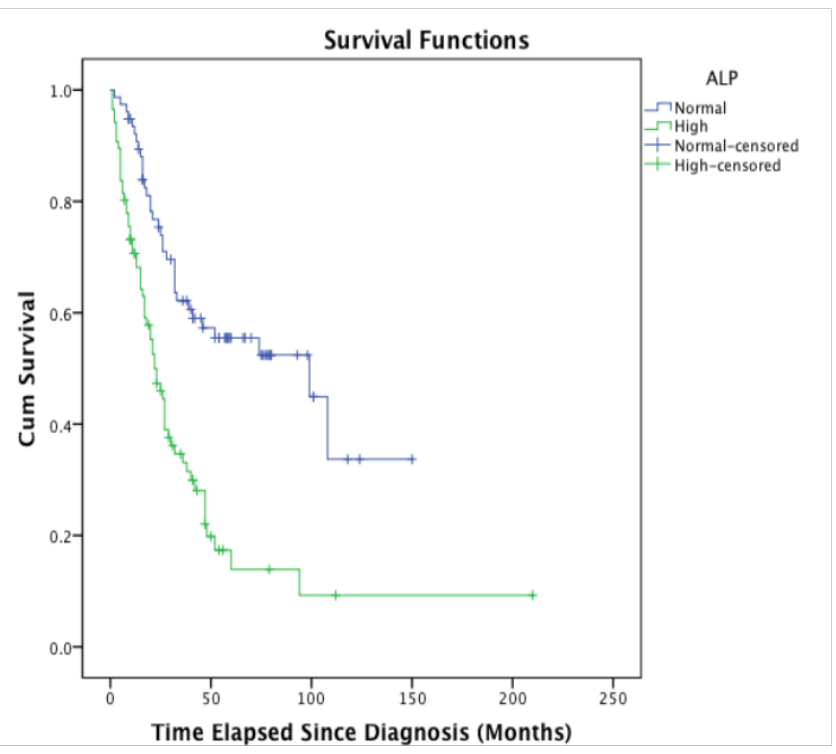

Figure 3 Overall survival of Osteosarcoma with high ALP.

The presence of lung metastasis significantly affects the survival in our group of osteosarcoma patients. They carried poor survival results. However, the timing of appearance of the pulmonary metastasis is not significant.

Our study sample had 90 patients (55.2\%) who developed lung metastases occurring at different stages of the disease; while the remaining 73 patients $(45.8 \%)$ had localised disease. In terms of survival, the metastatic group had a survival rate of $37 \%$ at 5 years; whereas in the non-metastatic group, the survival rate is $85 \%$ at 5 years. There was a highly significant statistical difference between those who had localised disease and those who had metastatic disease. In our group of patients with pulmonary metastasis, their survival rate is comparable to a few studies. ${ }^{15,16}$ In our group of patients with localised disease, their survival rates were similarly observed by other authors. ${ }^{16-19}$

There were 121(74.2\%) patients who underwent surgical resection of the primary tumour, compared to $42(25.8 \%)$ patients who did not undergo any surgical procedures. The 2-year and 5-year survival rates for patients who underwent surgery were at $74 \%$ and $63 \%$, respectively. On the contrary, 2-year and 5-year survival rates for patients who didn't undergo any surgical intervention were $44 \%$ and $0 \%$ since none of these patients survived after 5 years. The comparison tests indicated that there is a high, statistically significant difference between these patients.

Pertaining to the survival rates of those who had surgery, the group of patients who underwent limb salvage surgery (LSS) had their 2 -year \& 5-year survival rates at $82 \%$ and $75 \%$, respectively.

On the other hand, the limb-amputation group's 2-year and 5-year survival rates were at $54 \%$ and $22 \%$. The comparison tests show that there is a high, statistically significant difference between these two groups.

In our study, most patients had undergone limb-salvage surgery instead of amputation. The site of tumour, the involvement of the neurovascular bundles and the presence or absence of a pathologic fracture determined the choice of surgical procedure. The group of patients who had limb-amputation were mainly due to the following reasons: the initial tumour mass was massive (with or without concomitant lung metastasis), the involvement or proximity of the neurovascular bundles or those who had rapid progression of the tumour (such as in chemotherapy non-responders). In our study, the LSS group had a superiorly significant survival outcome when compared to the limb-amputation group.

Our study further confirmed the findings from a meta-analysis in 2014, which concluded high LDH as a poor prognostic indicator. ${ }^{8}$ There were 10 studies with the range of 28 to 198 patients per study. However, only five of the ten studies identified high serum LDH level as an indicator of poor prognosis, while the remaining five studies presented no statistical impact of high serum LDH level on the survival rate. ${ }^{8,18-23}$

\section{Conclusion}

In this study, prognostic factors that significantly predict the outcome of the overall survival by univariate analysis included metastatic disease at presentation, surgical intervention, high LDH and high ALP. LDH and surgery possessed significant values on the multiple variable analysis.

We concluded that pre-treatment serum LDH and ALP have a significant influence on the final survival rate. Close follow-ups and regular evaluations are crucial for this group of patients.

\section{Acknowledgements}

None.

\section{Conflict of interest}

The author declared no potential conflict of interest with respect to the research authorship, and or publication of the article.

\section{References}

1. Mirabello L, Troisi R J, Savage S A. Osteosarcoma incidence and survival rates from 1973 to 2004. Cancer. 2009;115(7):1531-1543.

2. Puri A, Jaffe N, Gelderblom H. Editorial Osteosarcoma: Lessons Learned and Future Avenues. Sarcoma. 2013;1-3.

3. Bielack SS, Kempf-Bielack B, Delling G, et al. Prognostic factors in high-grade osteosarcoma of the extremities or trunk: an analysis of 1,702 patients treated on neoadjuvant cooperative osteosarcoma study group protocols. J Clin Oncol. 2002;20(3):776-90.

4. Bramer JA, Abudu AA, Tillman RM, et al. Pre-and post-chemotherapy alkaline phosphatase levels as prognostic indicators in adults with localised osteosarcoma. European Journal of Cancer. 2005;41:28462852.

5. Jyoti Bajpai, Ajay Puri, Kajal Shah, et al. Chemotherapy Compliance in Patients with Osteosarcoma. Paediatric blood \& cancer. 2013;60(1):4044.

6. Faisham WI, Mat Saad AZ, Alsaigh LN, et al. Prognostic factors and survival rate of osteosarcoma: A single-institution study. Asia Pacific Journal of Clinical Oncology. 2015; 13(2):e104-e110.

7. Davis A M, Bell R S, Goodwin P J. Prognostic Factors in Osteosarcoma a Critical-Review. Journal of Clinical Oncology. 1994;12:423-431. 
8. Chen J1, Sun MX, Hua YQ, et al. Prognostic significance of serum lactate dehydrogenase level in osteosarcoma: a meta-analysis. J Cancer Res Clin Oncol. 2014;140:1205-1210.

9. Bramer JA, van Linge JH, Grimer RJ, et al. Prognostic factors in localized extremity osteosarcoma: A systematic review. Eur J Surg Oncol. 2009;35(10):1030-1036.

10. Pakos EE, Nearchou AD, Grimer RJ, et al. Prognostic factors and outcomes for osteosarcoma: An international collaboration. Eur J Cancer. 2009;45(13):2367-2375.

11. Munajat I, Zulmi W, NorazmanMZ, et al. Tumour volume and lung metastasis in patients with osteosarcoma. J Orthop Surg (Hong Kong). 2008;16:182-185.

12. Bacci G, Ferrari S, Bertoni F, et al. Long-term outcome for patients with nonmetastatic osteosarcoma of the extremity treated at the istitutoortopedicorizzoli according to the istitutoortopedicorizzoli/ osteosarcoma-2 protocol: an updated report. $J$ Clin Oncol. 2000;18(24):4016- 27.

13. Bacci G, Longhi A, Ferrari S, et al. Prognostic significance of serum lactate dehydrogenase in osteosarcoma of the extremity: experience at Rizzoli on 1421 patients treated over the last 30 years. Tumori. 2004;90(5):478-484.

14. Bacci G, Picci P, Ferrari S, et al. Prognostic-Significance of Serum Alkaline-Phosphatase Measurements in Patients with Osteosarcoma Treated with Adjuvant or Neoadjuvant Chemotherapy. Cancer. 1993;71(4):1224-1230.

15. Harting MT, Blakely ML, Jaffe N, et al. Long-term survival after aggressive resection of pulmonary metastases among children and adolescents with osteosarcoma. Journal of Paediatric Surgery. 2006;41(1):194-199.
16. Aljubran AH, Griffin A, Pintilie M, et al. Osteosarcoma in adolescents and adults: survival analysis with and without lung metastases. Ann Oncol. 2009;20(6):1136-1141.

17. Hegyi M, Semsei AF, Jakab Z, et al. Good Prognosis of Localized Osteosarcoma in Young Patients Treated with Limb-Salvage Surgery and Chemotherapy. Pediatr Blood Cancer. 2011;57(3):415-422.

18. Wu PK, Chen WM, Chen CF, et al. Primary osteogenic sarcoma with pulmonary metastasis: clinical results and prognostic factors in 91 patients. Jpn J Clin Oncol. 2009;39(8):514-522.

19. Meyers PA, Heller G, Healey JH, et al. Osteogenic-Sarcoma with Clinically Detectable Metastasis at Initial Presentation. J Clin Oncol. 1993;11(3):449-453.

20. Gonzalez-Billalabeitia E, Hitt R, Fernandez J, et al. Pre-treatment serum lactate dehydrogenase level is an important prognostic factor in highgrade extremity osteosarcoma. Clin Transl Oncol. 2009;11(7):479-483.

21. Pochanugool 1, Subhadharaphandou T, Dhanachai M, et al. Prognostic factors among 130 patients with osteosarcoma. Clin Orthop Relat Res. 1997;(345):206-214.

22. Aparicio J, Segura A, Montalar J, et al. Long-term results after combined modality treatment for non-metastatic osteosarcoma. Med Oncol. 1999;16(4):255-260.

23. Hagleitner MM, Hoogerbrugge PM, van der Graaf WT, et al. Age as prognostic factor in patients with osteosarcoma. Bone. 2011;49(6):11737. 